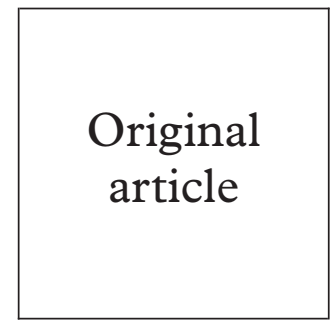

\title{
Are adolescents being screened for sexually transmitted diseases? A study of low income African American adolescents in San Francisco
}

\author{
J M Ellen, M A Lane, J McCright
}

\begin{abstract}
Objectives: To determine the proportion of sexually experienced African American adolescents who report having been screened for sexually transmitted diseased (STDs), and to determine the proportion who report having been screened for STDs among those adolescents who have had a preventive primary healthcare visit in the past 2 years.

Methods: A telephone survey of a population based sample of African American 12-17 year old adolescents residing in a low income San Francisco neighbourhood with a high prevalence of STDs.

Results: Of the 302 adolescents surveyed, 118 (39\%) reported a history of sexual intercourse. Of these, $26 \%$ of the males and $59 \%$ of the females had been screened for an STD in the previous 12 months. $31 \%$ of the males and $63 \%$ of the females had been screened for an STD in the previous 24 months. Of the 93 participants who had had a preventive primary care visit since their first episode of sexual intercourse, $26 \%$ of the males and $60 \%$ of the females had been screened for an STD in the previous 24 months.

Conclusions: Sexually experienced African American adolescents in San Francisco are being screened for STDs at rates well below that recommended by current clinical guidelines. A low rate of screening was found even in those adolescents who had been seen for a preventive primary care visit since they first had sex. This suggests that the preventive primary care visit is not being used to its full potential as an opportunity to screen and treat adolescents for STDs. Capitalising on this opportunity to screen may increase the number of STDs diagnosed and, thus, decrease rates of STDs in this population.
\end{abstract}

(Sex Transm Inf 2000;76:94-97)

Keywords: sexually transmitted diseases; adolescents; screening; San Francisco

\section{Introduction}

A cornerstone of STD prevention and control is the early diagnosis and treatment of bacterial STDs. Researchers have shown that testing individuals for STDs, even in the absence of symptoms, can prevent negative health outcomes. ${ }^{1}$ Accordingly, most current practice guidelines recommend that clinicians test those adolescents who have had sexual intercourse for STDs at least annually as part of a primary preventive health visit - that is, during a routine periodic history and physical examination..$^{2-4}$ This testing in the absence of symptoms is commonly known as screening. The recent Institute of Medicine report, "The Hidden Epidemic: Confronting Sexually Transmitted Diseases," suggests that the integration of STD screening into primary care visits will increase the number of adolescents who are screened for STDs, and thus, would increase the number of adolescents diagnosed and treated for STDs.

There are few data on how many American adolescents who have had sexual intercourse are screened for STDs. A 1988 US survey showed that $46 \%$ of sexually experienced 15-19 year old African American women and $34 \%$ of all sexually experienced $15-19$ year old women had been tested for STDs in the 12 months before the survey. ${ }^{6}$ The researchers found that women were more likely to have been tested for STDs if they had had any of the following: a family planning visit in the 12 months before the survey, a higher number of sexual partners, a recent pregnancy, or a history of a STD. However, since this study did not differentiate between times when women were tested for an STD because of symptoms and times when they were screened in the absence of symptoms, it is difficult to assess the role of primary care in the secondary prevention of STDs. The study also did not include men.

Another study that examined STD screening practices for adolescents reported on laboratory tests done on patients who were enrolled in seven health maintenance organisations (HMOs) in Massachusetts in 1992. ${ }^{7}$ The investigators estimated that $21 \%$ of the $15-19$ year old sexually experienced women enrolled in the HMOs were tested for STDs. Interestingly, they arrived at this estimate without knowing how many of the adolescents enrolled in the HMOs had a history of sexual intercourse-that is, the number of women who should have been screened. Rather, they had to use data from another study of high school students from Massachusetts to infer the proportion of female adolescents enrolled in the HMOs likely to have a history of sexual intercourse. This methodology calls into question the reliability of their estimate and, like the previous study, this one did not differentiate between whether women had symptomatic 
testing or asymptomatic screening, did not determine the number of adolescent women who had received preventive primary care, and did not include adolescent men.

The limitations of these two studies demonstrate how difficult it is to assess whether adolescents are being appropriately screened for STDs. To answer this question properly requires that a community based sample of adolescents answer detailed questions about their sexual histories in order to determine which adolescents are sexually experienced, which have had a preventive primary care visit, and which had STD symptoms at the time of the visit.

The objective of this population based study of African American adolescents was to determine the proportion of sexually experienced African American adolescents who report having been screened for STDs. In addition, we wanted to determine the proportion of sexually experienced adolescents who had been screened for STDs among those adolescents who had attended a primary preventive health visit in the past 2 years. We focused on African American adolescents because the burden of disease is highest in this population. For example, the 1997 reported rate of chlamydia in American adolescents aged 15-19 years was 1126 per 100 000. The reported rate in African American males was more than 12 times, and in African American females was more than six times, the corresponding rates in age matched white adolescents. ${ }^{8}$

\section{Methods}

This analysis was part of a larger study that examined health seeking behaviour by African American adolescents and was used to inform the development of a community based intervention to reduce STDs in these youths. Target participants were African American adolescents between the ages of 12 and 17 years who lived in a predominantly low income, African American neighbourhood in San Francisco. The prevalence of reportable STDs in the neighbourhood is the highest in the city (San Francisco Department of Public Health, unpublished data).

Between November 1996 and March 1997, we contacted a sample of households using a list assisted random digit set of telephone numbers from three different prefixes. More than $98 \%$ of households in the neighbourhood are estimated to have a telephone service and $65 \%$ of these households are served by the three telephone prefixes we used. Potential participants were contacted in the evenings and at weekends. If more than one adolescent lived in a household, each was considered eligible to participate. Only adolescents who were identified by their parent or guardian as being African American were enrolled in the study. Verbal informed consent was obtained from both the parent and the adolescent. After the 30 minute telephone interview, adolescents were mailed a $\$ 10.00$ cheque. The study was conducted with institutional review board approval.
In all, we contacted 285 households in which 394 eligible adolescents lived. Participation was declined by $13.7 \%$ of parents or guardians. A further $9.6 \%$ of adolescents either declined consent or were unavailable for interview. There was no difference in age or sex of participants and non-participants. Thus, the sample consisted of 302 participants who lived in 224 households and represented $77 \%$ of eligible participants.

Wording of survey items was based upon results of focus groups and pilot tests. Most questions were worded such that adolescents could respond with "yes" or "no" or with a number to maintain the confidentiality of their responses from family members. Whenever possible, participants were interviewed while they were alone in the room. Less than $10 \%$ reported that a parent or guardian or other adult was in the same room at any time during the interview.

Basic demographic data (age, sex, and educational level of parents) were collected from each adolescent. Health insurance data were collected from the parent or guardian. Participants were asked a variety of questions about their general health seeking behaviour, including the names of clinics they had attended. We determined whether participants had had a preventive primary care visit by their response to the question: "When was the last time you had a full physical? By a 'full physical,' we mean a visit where they examined your whole body and asked you a lot of questions about your health and things that might affect your health?" Participants were then asked whether they had ever had sexual intercourse. This was defined for them as " 'having sex,' or 'going all the way,' like where a boy puts his penis in a girl's vagina." Participants who reported a history of sexual intercourse were then asked when they had first had sex and the number of sexual partners they had had in their lifetime. They were then asked if they had ever been checked for an STD when they had symptoms, and when that was. Symptoms were described to them as including times when they had pain or discharge from their penis or vagina that was not urine. Later in the interview, participants were asked if they had ever been checked for an STD when they did not have any symptoms, and when that was. In addition, they were asked if they had ever been diagnosed with, or treated for, any of the following STDs: genital herpes, gonorrhoea, chlamydia, pelvic inflammatory disease, and syphilis.

We defined an adolescent as having had a preventive primary care visit if the visit had occurred within the previous 2 years. We determined which participants had a history of sexual intercourse at the time of their primary care visit based on the responses of participants to the questions regarding when they had first had sex and when they last had a preventive primary care visit. In order to assess the influence of the non-independence of participants (many of the participants resided in households where another participant resided), we reanalysed our data including only one partici- 
Table 1 Characteristics of sexually experienced male and female African American adolescent participants, San Francisco, $1997(n=118)$

\begin{tabular}{lll}
\hline & $\begin{array}{l}\text { Men } \\
(n=65) \%\end{array}$ & $\begin{array}{l}\text { Women } \\
(n=53) \%\end{array}$ \\
\hline Age (years) & & \\
$12-14$ & 22.6 & 17.5 \\
15 & 17.0 & 12.5 \\
16 & 20.8 & 35.0 \\
17 & 39.6 & 35.0 \\
Maternal education* & & \\
$\quad$ Less than high school & 15.1 & 15.0 \\
High School & 32.1 & 50.0 \\
$\quad$ More than high school & 52.8 & 35.5 \\
History of at least one STD & & \\
$\quad$ Yes & 11.3 & 17.5 \\
$\quad$ No & 88.7 & 82.5 \\
Lifetime number of sex partners & & \\
$\quad$ 1 & 23.1 & 60.0 \\
2 & 15.4 & 10.0 \\
$\geqslant 4$ & 21.2 & 7.5 \\
& 40.4 & 22.5 \\
\hline
\end{tabular}

$\star$ Paternal education was substituted for maternal education for participants who did not live with their mother or a female guardian.

†Ever diagnosed with or treated for chlamydia, gonorrhoea, syphilis, pelvic inflammatory disease, or genital herpes.

pant per household. There was no clinically significant difference between our initial and revised results. Thus, we have presented our initial results.

\section{Results}

Of the 302 adolescents surveyed, 118 (39\%) reported at least one episode of sexual intercourse. Selected characteristics of these 118 adolescents are shown in table 1. Seventeen of the male participants $(26.2 \%)$ and 31 of the female participants $(58.5 \%)$ had been screened for STDs in the previous 12 months. Twenty of the male participants $(31.3 \%)$ and 32 of the female participants $(60.4 \%)$ had been screened for STDs in the previous 24 months. In order to control for the timing of first sexual intercourse, we examined the screening rates among male $(n=41)$ and female participants $(n=20)$ who had first engaged in sex 2 or more years before the interview and among male $(n=23)$ and female participants $(n=32)$ who had first engaged in sex less than 2 years before the interview. For male participants, there was no difference in the 2 year screening rate between those with less recent and those with more recent first sex $(30.4 \% v 31.7 \%$; $=0.8)$. However, for female participants, there was a difference in the 2 year screening rate between those with less recent and those with more recent first $\operatorname{sex}(80.0 \% v 50.0 \%$; $\mathrm{p}<0.05)$.

To answer the question of whether adolescents who are being seen for preventive primary care are also being screened for STDs, we focused on the 53 male participants and 40 female participants who had a preventive primary care visit after they first had sex. As a group, these adolescents reported receiving primary care at several different types of clinics, including both private and publicly funded sites. Among those adolescents who had attended a primary preventive care visit, 14 of the male participants $(26.4 \%)$ and 24 of the female participants $(60.0 \%)$ had been screened for STDs at some time in the previous 24 months. Table 2 shows the percentage of
Table 2 African American adolescents who had a primary preventive health visit in past two years and were screened for an STD according to selected STD risk factors, San Francisco, 1997

\begin{tabular}{|c|c|c|c|c|}
\hline & \multicolumn{2}{|c|}{ Males $(n=53)$} & \multicolumn{2}{|c|}{ Females $(n=40)$} \\
\hline & No & $\%$ Screened & No & $\%$ Screened \\
\hline \multicolumn{5}{|c|}{ Age (years) } \\
\hline $12-14$ & 12 & 16.7 & 7 & 28.6 \\
\hline 15 & 9 & 44.4 & 5 & 80.0 \\
\hline 16 & 11 & 27.3 & 14 & 50.0 \\
\hline 17 & 21 & 23.8 & 14 & 78.6 \\
\hline \multicolumn{5}{|c|}{ History of at least one $\mathrm{STD}^{\star}$} \\
\hline Yes & 6 & 33.3 & 7 & 71.4 \\
\hline No & 47 & 25.5 & 33 & 57.6 \\
\hline \multicolumn{5}{|c|}{ Lifetime number of sex partners } \\
\hline 1 & 12 & 41.7 & 24 & 54.2 \\
\hline 2 & 8 & 12.5 & 4 & 75.0 \\
\hline 3 & 11 & 36.4 & 3 & 33.3 \\
\hline$\geqslant 4$ & 21 & 19.1 & 9 & 77.8 \\
\hline
\end{tabular}

${ }^{\star}$ Ever diagnosed with or treated for chlamydia, gonorrhoea, syphilis, pelvic inflammatory disease, or genital herpes.

participants screened according to selected STD risk factors. Eleven of the male participants $(20 \%)$ and 16 of the female participants $(40 \%)$ were screened for STDs during the primary preventive care visit. The small number of sexually experienced adolescents who had received a preventive primary care visit precluded us from using inferential statistics to test for differences in screening rates according to STD risk factors.

\section{Discussion}

The results of our study indicate that sexually experienced African American adolescents living in a low income area of San Francisco are being screened for STDs at rates below that recommended by current practice guidelines. Even those adolescents who receive primary health care are not being screened at the recommended level. These findings are of particular concern because the adolescents in our study are among the highest risk group for STDs in the United States. They are low income African American adolescents who live in a neighbourhood with a high prevalence of STDs.

The low rates of screening for STDs may be related to any of the potential hurdles that need to be crossed before an STD screen occurs. Firstly, clinicians need to take a sexual history. One study found that approximately $75 \%$ of Californian physicians report that they routinely take a basic sexual history from adolescents, ${ }^{9}$ while another found that only $40 \%$ report doing so. ${ }^{10}$ In some cases, adolescents may be unwilling to disclose the truth, particularly if the physician does not make assurances about protecting confidentiality. ${ }^{11} \mathrm{It}$ is also possible that even if a physician determines that an adolescent is sexually experienced, the physicians may still not perform an STD screen owing to a lack of awareness of current screening guidelines ${ }^{12}$ or a poor sense of competence to perform an STD screen. ${ }^{13}$ In some cases, adolescents may refuse to be screened for STDs.

The methodology of our study has limitations. It is possible that adolescents may not have been honest in their responses about their sexual behaviour and their STD history 
because of the sensitivity of the data. ${ }^{14} 15$ Because of this concern, our interviewers were careful to reassure adolescents that all responses were confidential. Also, all questions were pilot tested and were worded such that they could be answered with only a "yes" or "no" or with a number to prevent a household member from understanding an adolescent's responses. Despite these efforts, adolescents may have overreported or underreported their sexual behaviour and STD history. ${ }^{14} 15$

It is also possible that some male adolescents were screened for an STD by leucocyte esterase testing of urine without being aware of it, thus falsely lowering self reported rates of STD screening. ${ }^{16}$ This scenario is not likely to be true for female adolescents. Since urine based screening of women was not routinely available at the time of the survey, it is most likely that an adolescent female would have realised that she had an STD screen because it required a pelvic examination. But it is possible that an adolescent female thought she had been tested for STDs when she had not. For example, the clinician may have only collected a cervical smear for a Papanicolaou test but the female adolescent thought that she had had an STD screen. Thus, female adolescents may have overreported being screened for STDs.

A further limitation of our study is that we surveyed only African American adolescents who live in a small geographic area. Certainly, it would be ideal to know the proportion of all American adolescents that is being screened for STDs. However, in the absence of data from a nationally representative sample, we believe it is informative to collect data from smaller populations of adolescents, and in particular, those who are at highest risk for acquiring an STD.

In summary, African American adolescents are being screened for STDs at a rate below that recommended by current practice guidelines. The preventive primary care visit is not being used to its full potential as a valuable opportunity to screen adolescents for STDs. Capitalising on this opportunity may increase rates of STD screening and, thus, decrease rates of STDs in adolescents. One hope is that recent technological advances in the diagnosis of STDs - that is, urine based STD testing, may increase both clinicians' willingness to screen and adolescents' willingness to be screened for STDs, and, thereby, increase the number of adolescents screened for STDs. The research was supported, in part, by the Centers for Disease Control and Prevention, (Accelerated Prevention Campaign, Enhanced Projects for STD Prevention in High Risk Youth, Grant No H25/CCH-904371), National Institute of Allergy an Infectious Diseases (grant no R01-AI36986), and Maternal and Child Health Bureau, Health Resources and Services Administration (grant no MCJ000978). We would like to dedicate this manuscript to the memory of Karen Garrett whose commitment to the implementation of the project ensured its success.

1 Scholes D, Stergachis A, Heidrich F, et al. Prevention of pelvic inflammatory disease by screening for cervical chlamydial infection. N Engl f Med 1996;334:1362-6.

2 Elster AB, Kuszets N. Guidelines for adolescent preventive services (GAPS). Baltimore, MD: Williams and Williams, 1993.

3 Green M (Ed). Bright futures: guidelines for health supervision of infants, children, and adolescents. Arlington, VA: Nationa Center for Education in Maternal and Child Health, 1994

4 Clinician's handbook of preventive services. 2nd ed. Put prevention into practice. US Department of Health and Human Services, Public Health Service, Office of Public Health and Sci, Public motion. Rockville, MD: ODPHP, 1998.

5 Institute of Medicine (US) Committee on Prevention and Control of Sexually Transmitted Diseases. In: Eng T, Butler W, eds. The hidden epidemic: confronting sexually transmitler W, eds. The hidden epidemic: confronting sexually transmit-
ted diseases. Washington, DC: National Academy Press, ted diseas.

6 Mosher WD, Aral SO. Testing for sexually transmitted disof reproductive age: United States, 1988. Family Planning Perspectives 1991;23:216-21.

7 Thrall JS, McCloskey L, Spivak H, et al. Performance of Massachusetts HMOs in providing pap smear and STD screening to adolescent females. $\mathcal{F}$ Adolesc Health 1998;22 $184-9$.

8 Division of STD Prevention. Sexually transmitted disease surveillance, 1997. US Department of Health and Human Services, Public Health Service. Atlanta: Centers for Disease Control and Prevention (CDC), September 1998.

9 Ellen JM, Franzgrote M, Irwin CEI Jr, et al. Primary care physicians' screening of adolescent patients: a survey of physicians' screening of adolescent patients: a survey

10 Millstein SG, Igra V, Gans J. Delivery of STD/HIV prevenMillstein SG, Igra V, Gans J. Delivery of STD/HIV preven-
tive services to adolescents by primary care physicians. $\mathcal{F}$ Adolesc Health 1996;19:249-57.

11 Ford CA, Millstein SG, Halpern-Felsher BL, et al. Influence of physician confidentiality assurances on adolescents' willingness to disclose information and seek future health care. ҰAMA 1997;278:1029-34

12 Christakis DA, Rivara FP. Pediatricians' awareness and attitudes about four clinical practice guidelines. Pediatric 1998;101:825-30.

13 Blum RW, Bearinger LH. Knowledge and attitudes of health professionals toward adolescent health care. If Adolesc Health Care 1990;11:289-94.

14 Rosenthal SL, Burklow KA, Biro FM, et al. The reliability of high risk adolescent girls report of their sexual history. $f$ Pigh risk adolescent girls report of

15 McHugh MT, Palusi VJ. Assessing prior history of sexually transmitted disease. $\mathscr{F} A M A$ 1992;267:1610-11.

16 Shafer MA, Schachter J, Moscicki AB, et al. Urinary leukocyte esterase screening test for asymptomatic chlamydial and gonococcal infections in males. $\mathcal{F} A M A$ 1989;262 2562-6. 